Article

\title{
Diverse and Abundant Secondary Metabolism Biosynthetic Gene Clusters in the Genomes of Marine Sponge Derived Streptomyces spp. Isolates
}

\author{
Stephen A. Jackson ${ }^{1,+} \mathbb{D}^{\mathbb{D}}$, Lisa Crossman ${ }^{2,3,+}{ }^{\dagger}$ Eduardo L. Almeida ${ }^{1}$, Lekha Menon Margassery ${ }^{1}$, \\ Jonathan Kennedy ${ }^{4}$ and Alan D.W. Dobson ${ }^{1,5, *(D)}$ \\ 1 School of Microbiology, University College Cork, National University of Ireland, T12 YN60 Cork, Ireland; \\ sjackson@ucc.ie (S.A.J.); lekha513@gmail.com (E.L.A.); edleaoalmeida@gmail.com (L.M.M.) \\ 2 School of Biological Sciences, University of East Anglia, Norwich Research Park, Norwich NR4 7TJ, UK; \\ L.Crossman@uea.ac.uk \\ 3 SequenceAnalysis.co.uk, NRP Innovation Centre, Norwich NR4 7UG, UK \\ 4 Invista Performance Technologies, The Wilton Centre, Wilton, Redcar, Cleveland TS10 4RF, UK; \\ jonathan.kennedy@invista.com \\ 5 Environmental Research Institute, University College Cork, National University of Ireland, Lee Road, \\ T23 XE10 Cork, Ireland \\ * Correspondence: a.dobson@ucc.ie; Tel.: +353-21-490-2743 \\ + These authors contributed equally to this work.
}

Received: 21 December 2017; Accepted: 16 February 2018; Published: 20 February 2018

\begin{abstract}
The genus Streptomyces produces secondary metabolic compounds that are rich in biological activity. Many of these compounds are genetically encoded by large secondary metabolism biosynthetic gene clusters (smBGCs) such as polyketide synthases (PKS) and non-ribosomal peptide synthetases (NRPS) which are modular and can be highly repetitive. Due to the repeats, these gene clusters can be difficult to resolve using short read next generation datasets and are often quite poorly predicted using standard approaches. We have sequenced the genomes of 13 Streptomyces spp. strains isolated from shallow water and deep-sea sponges that display antimicrobial activities against a number of clinically relevant bacterial and yeast species. Draft genomes have been assembled and smBGCs have been identified using the antiSMASH (antibiotics and Secondary Metabolite Analysis Shell) web platform. We have compared the smBGCs amongst strains in the search for novel sequences conferring the potential to produce novel bioactive secondary metabolites. The strains in this study recruit to four distinct clades within the genus Streptomyces. The marine strains host abundant smBGCs which encode polyketides, NRPS, siderophores, bacteriocins and lantipeptides. The deep-sea strains appear to be enriched with gene clusters encoding NRPS. Marine adaptations are evident in the sponge-derived strains which are enriched for genes involved in the biosynthesis and transport of compatible solutes and for heat-shock proteins. Streptomyces spp. from marine environments are a promising source of novel bioactive secondary metabolites as the abundance and diversity of smBGCs show high degrees of novelty. Sponge derived Streptomyces spp. isolates appear to display genomic adaptations to marine living when compared to terrestrial strains.
\end{abstract}

Keywords: marine Streptomyces; biosynthetic gene clusters; secondary metabolites

\section{Introduction}

Microbially derived natural products are an important source of novel biotherapeutic agents, with more than 22,000 biologically active compounds being isolated to date from microorganisms [1]. Approximately $45 \%$ of these are produced by Actinobacteria, with members of the genus Streptomyces being particularly proficient producers [2]. Actinomycetes have produced a number of important drug 
leads which have subsequently been developed into immunosuppressive (rapamycin), anti-cancer (bleomycin, daunorubicin, mitomycin) and anti-microbial (amphotericin B, erythromycin, vancomycin) drugs. However, there is an ongoing need for novel bioactive compounds, particularly antibiotics, due to the emergence of antibiotic resistance in clinically important bacterial pathogens [3]. The rapid dissemination of antibiotic resistances has coincided with the antibiotic "discovery void" where no new antibiotic scaffolds have been identified since 1987 [4].

Strategies to address the dearth of newly identified clinical therapeutics have progressed through extensive screening regimes of environmental microbial isolates [5], through the dawn of metagenomics where environmental DNA was cloned to heterologous expression hosts [6]. This promised to allow access to the genetic repertoires of uncultivable microbes but resulted in the "great screen anomaly" [7] where gene and product discovery from clone libraries is disappointingly low compared to what might have been expected. The trend has now returned to investigating cultivable isolates, armed with powerful genome sequencing technologies which have made huge numbers of draft and completely resolved bacterial genomes available for "genome mining". The somewhat surprising outcome of these efforts is that the genomes of, for example, Actinobacteria contain dozens of secondary metabolism biosynthetic gene clusters (smBGCs), the majority of which appear to be "silent" or "cryptic" [8]. This discovery led to the estimate that no more than $10 \%$ of the genes encoding small molecules in bacteria have to date been identified and furthermore that a single gene cluster can give rise to a suite of related compounds which can act synergistically to inhibit bacterial growth [9]. Further conservative estimates derived from genome sequence data suggest that the genus Streptomyces alone may be capable of producing 150,000 secondary metabolites with fewer than $5 \%$ of these having been characterized to date [10].

These findings have focused efforts to attempt to activate these silent clusters through imaginative approaches such as OSMAC (one strain many compounds) cultivation efforts, co-cultivation of microbes to provide environmental cues or stresses, using genome data to 'awaken' gene cluster activators, to delete suppressors or to engineer strong promoters upstream of BGCs of interest [11]. A high-throughput approach, termed high-throughput elicitor screens (HiTES), has been developed where reporter genes such as lacZ or GFP are cloned into a BGC of interest and the strain is exposed to libraries of small compound potential 'elicitors' such as sub-inhibitory concentrations of antibiotics [12]. The elegance of this approach is that endogenous regulation of gene expression is maintained, the reporter genes allow the identification of the previously silent clusters, the gene cluster activator is identified, and the small molecule gene cluster product is also identified. This approach allowed $\mathrm{Xu}$ and colleagues to identify two cytotoxins as inducers of BGCs in Streptomyces albus J1074, and to characterize 14 novel small molecule products, one of which is a novel antifungal and another which may find use as an anti-cancer agent [13].

Genome mining of terrestrial actinomycete bacteria has proven to be useful in the identification of secondary metabolism biosynthetic gene clusters (smBGCs) such as those encoding the bottromycin, planosporicin and tunicamycin clusters [14] and it is clear that based on the successful genome mining on the Salinispora genus, which resulted in the identification of a number of novel biosynthetic gene clusters [15], and the recent work on the anthracimycin gene cluster from the marine derived Streptomyces sp. T676 [16] amongst others, that genome mining may also prove effective in the identification of potentially novel chemistry from other marine derived actinomycete bacteria.

Marine derived actinomycetes are known to produce a broad range of structurally complex secondary metabolites with broad biological activities, and they are a source of an ever increasing number of natural products $[17,18]$. Novel Actinomycetes, including novel Streptomycetes, have also been isolated from deep-sea environments. The deep-sea is a harsh environment with high pressures, low temperatures, high salinity and low light and oxygen concentrations [19]. Interestingly, Streptomycete-derived novel bioactive secondary metabolites have been identified from isolates from the deep-sea [19]. Antibacterial and cytotoxic metabolites, are produced from a type I PKS gene cluster by Streptomyces sp. SCSIO 01127 which was isolated from a depth of $1350 \mathrm{~m}$ in the South China Sea [20] 
while antibacterial compounds, are produced by Streptomyces scopuliridis SCSIO ZJ46 which was isolated from a depth of $3536 \mathrm{~m}$ in the South China Sea [21]. Streptomyces drowzdowiczii SCSIO 10141, isolated from $1396 \mathrm{~m}$ in the South China Sea is known to produce the anti-infective metabolites [22]; cytotoxic and antibacterial compounds are produced by another deep sea isolate, Streptomyces niveus SCSIO 3406 [23].

With this in mind and following an extensive screening regime of marine actinomycete bacteria which we had previously isolated from the shallow water sponge Haliclona simulans [24] and from two deep-sea sponges Lissodendoryx diversichela and Stelletta normanii [25], involving growth inhibition of a number of clinically relevant bacterial and fungal species, 13 marine sponge-derived Streptomyces spp. strains were chosen for whole genome sequencing in an attempt to determine the secondary metabolite biosynthetic potential of these strains.

\section{Results and Discussion}

\subsection{Antimicrobial Activities}

A total of over 540 actinomycetes, including some which had previously been isolated from shallow water and deep-sea sponges in Irish waters [24-26], were screened for growth inhibition of a number of clinically relevant bacterial and fungal/yeast species. Thirteen of these strains which displayed the most interesting range of bioactive antimicrobial activities, including growth inhibition of problematic anti-microbial resistant (AMR) human pathogens such as methicillin-resistant Staphylococcus aureus (MRSA) and vancomycin-intermediate Staphylococcus aureus (ViSA), were identified for subsequent analysis (Table 1).

Table 1. Antimicrobial activities of cultured sponge bacteria using deferred antagonism assays. ${ }^{1}$ Bacillus cereus FPL1; ${ }^{2}$ Bacillus subtilis $1 \mathrm{~A} 40 ;{ }^{3}$ B. subtilis 1E32; ${ }^{a}$ hVISA (Heterogonous Vancomycin Intermediate Staphylococcus aureus) 22900; b MRSA (Methicillin resistant S. aureus) ST544; C VISA (Vancomycin intermediate S. aureus) 35403; ' S. aureus NCIMB 9518: ' +' = positive. ' -' = negative; n.d. not determined. * adapted from Kennedy et al., 2009 [19].

\begin{tabular}{|c|c|c|c|c|c|c|c|}
\hline \multirow[b]{2}{*}{ Test Strain } & \multicolumn{2}{|c|}{ Gram Negative Bacteria } & \multicolumn{3}{|c|}{ Gram Positive Bacteria } & \multicolumn{2}{|c|}{ Yeasts } \\
\hline & $\begin{array}{c}\text { E. coli } \\
\text { NCIMB } 12210\end{array}$ & $\begin{array}{l}\text { P. aeruginosa } \\
\text { PAO1 }\end{array}$ & $\begin{array}{l}\text { Bacillus } \\
\text { pp. }\end{array}$ & $\begin{array}{l}\text { Staphylococcus } \\
\text { spp. }\end{array}$ & $\begin{array}{l}\text { L. monocyte } \\
\text { genes F } 2365\end{array}$ & $\begin{array}{l}\text { Candida } \\
\text { spp. }\end{array}$ & $\begin{array}{l}\text { A. fumigatus } \\
\text { ATCC } 46645\end{array}$ \\
\hline SM1* & + & - & $+1,2$ & $++^{a}$ & + & + & n.d. \\
\hline SM5 * & + & - & $+1,2$ & $++^{a}$ & - & - & n.d. \\
\hline SM9 * & + & - & - & - & - & - & n.d. \\
\hline SM10* & + & n.d. & - & - & - & - & n.d. \\
\hline SM11* & - & - & +2 & $+a, b$ & - & - & n.d. \\
\hline SM12* & - & + & - & $++^{a}$ & - & - & n.d. \\
\hline SM14* & - & - & $+1,2$ & $++^{a}$ & - & - & n.d. \\
\hline SM16 * & - & + & $+{ }^{2}$ & $+{ }^{c}$ & - & - & n.d. \\
\hline $\mathrm{SM} 17^{*}$ & + & - & - & $+b$ & - & + & n.d. \\
\hline SM18* & - & - & +2 & $+b$ & - & - & n.d. \\
\hline FMC008* & - & + & +2 & $+{ }^{d}$ & - & - & n.d. \\
\hline B226SN101 & - & - & -3 & - & n.d. & + & + \\
\hline B188M101 & - & - & $-{ }^{3}$ & - & n.d. & + & + \\
\hline
\end{tabular}

\subsection{Genome Sequencing}

The genomes of these 13 Streptomyces spp. were sequenced using Illumina MiSeq paired-end sequencing. These strains were designated as follows: SM1, SM5, SM9, SM10, SM11, SM12, SM14, SM16, SM17, SM18, FMC008 isolated from shallow water sponges and B188M101 and B226SN101, isolated from deep-sea sponges. Genome assemblies resulted in a large number of contigs $(n=195-1592)$ (Table 2). Gap closure of these assemblies was hampered by the typical high GC content of the genomes of Streptomyces spp., as well as the presence of very many highly repetitive sequences in gene 
clusters such as polyketide synthase (PKS) and nonribosomal peptide synthetases (NRPS) clusters. The genomes ranged in size from 6.41 to $8.44 \mathrm{Mb}$ (Table 2).

Table 2. No. of contigs and total genome size of marine Streptomyces spp. isolates.

\begin{tabular}{ccc}
\hline Isolate ID & No. of Contigs & Total Length $\mathbf{( M b )}$ \\
\hline B188SM101 & 609 & 8.23 \\
B226SN101 & 580 & 8.39 \\
SM5 & 469 & 7.62 \\
SM10 & 195 & 7.48 \\
SM11 & 311 & 8 \\
SM12 & 910 & 6.5 \\
SM16 & 388 & 8.44 \\
SM1 & 1057 & 8.08 \\
SM9 & 1592 & 6.47 \\
FMC008 & 1369 & 6.5 \\
SM18 & 403 & 7.6 \\
SM14 & 639 & 6.41 \\
SM17 & 674 & 7.106 \\
\hline
\end{tabular}

\subsection{Taxonomy and Phylogeny}

The phylogeny of the 13 marine Streptomyces was determined by analysis of the 16S rRNA genes (Figure 1A), and by Feature Frequency Profile (Ffp) [27] of the whole genomes (Figure 1B), together with genomic analysis using Kraken (Supplementary Table S1) [28]. In the latter analysis, greater than $75 \%$ of the sequence reads were assigned closely to a known species while three of the strains (SM1, SM12 and SM14) could only be assigned at the genus level (Table 3).

The strains recruited to four main groups, Group A (comprising S. griseus, S. fulvissimus as well as SM11 and SM16 and both of the deep sea strains, B188M101 and B226SN101), Group B (comprising S. albus, SM17, SM9 and FMC008), Group C (comprising S. sirex, SM18, SM5 and SM10) and Group D (comprising SM12 and SM14) (Figure 1B). Analysis of the 16S rRNA genes suggest that SM1 is closely related to the group D isolates in a polyphyletic clade which is a sister clade to the Group B isolates, however Ffp based phylogeny indicates that SM1 falls outside of that polyphyletic clade. 


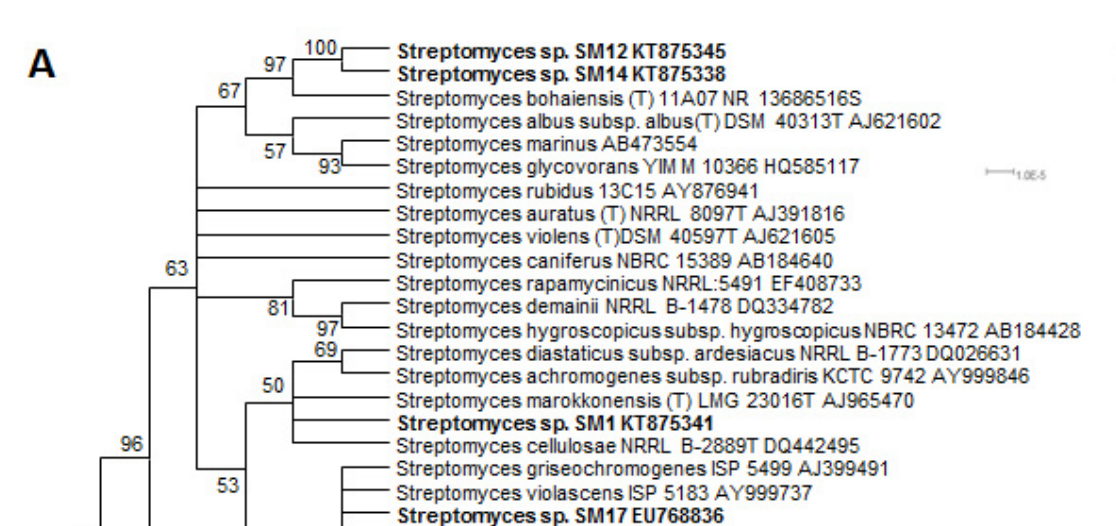

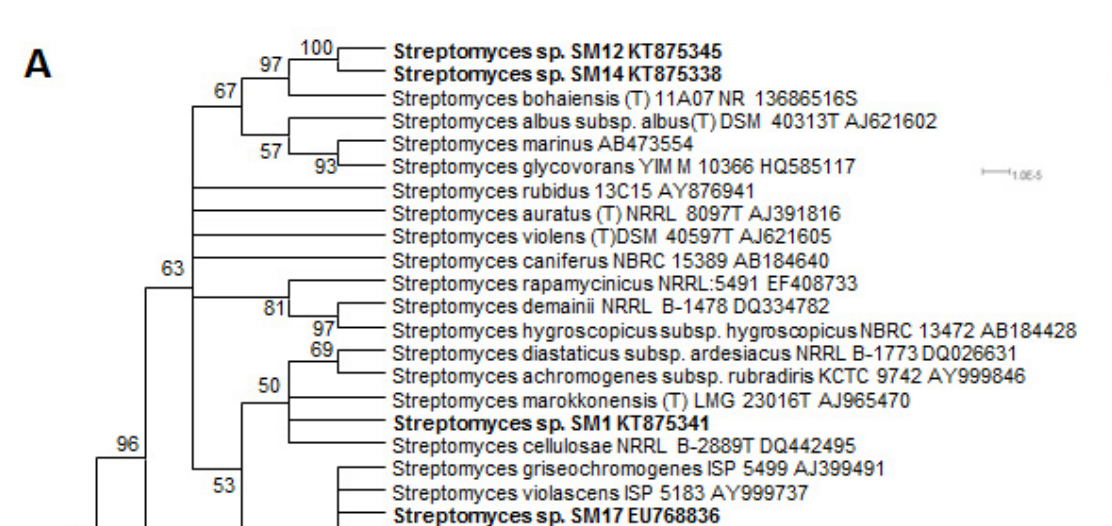

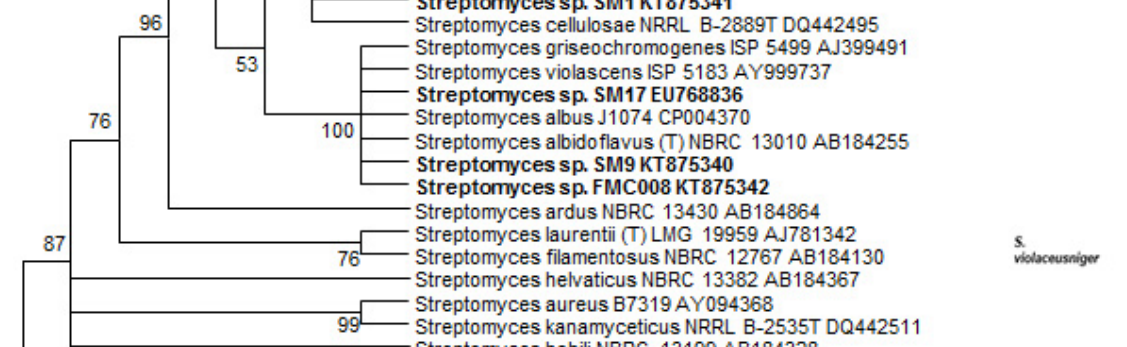
$99 \square$
Streptomyces aureus B7319 AY094368
Streptomyces kanamycticus NRRL B-2535T DQ442511
Streptomyces bobili NBRC 13199 AB 284328 Streptomyces yokosukanensis NRRL B-3353 DQ026652

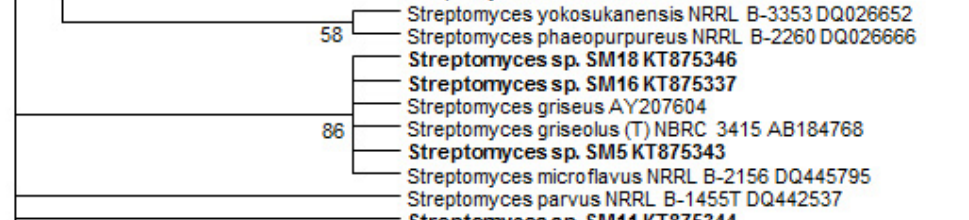
\begin{aligned} \hline \\ \hline $100 \square \\$\hline \\ Streptomyces atroolivaceus (T) LMG 19306 AJ781320 \\ Streptomyces sp. SM10 KT 875339 \end{aligned} Streptomyces setonii (T) ISP 5322 CSSP537 AY999784 Streptomyces sp. B188M101 KT875335 Streptomyces anulatus NRRL B-2000 Streptomyces pratensis (T) ch24J0806215

\section{B}

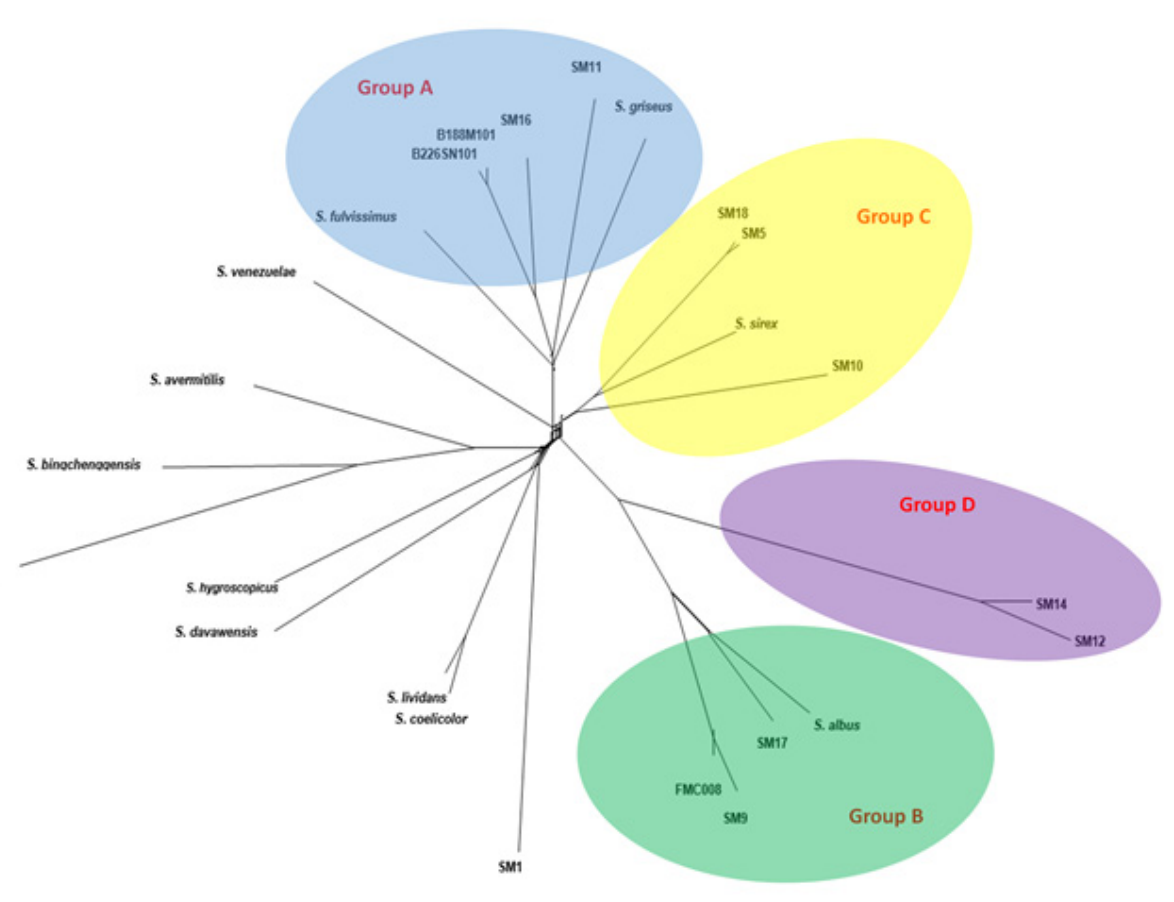

Figure 1. (A) 16S rRNA gene based (Streptomyces isolates from this study are in bold font) and (B) whole genome Frequency feature profile based phylogenetic trees of Streptomyces spp. marine isolates. 


\subsection{Pan Genome and Core Genome}

The pan genome (Figure 2A) of these marine isolates is open with an average of 648 genes added for each additional genome considered and a total gene complement of 14,066 genes shared amongst the isolates. The core genome (Figure 2B) comprises 1699 genes indicating that only $12 \%$ of the pan genome is shared by all of the strains examined here. The pan genome analysis highlights the genetic diversity within the genus, indicating that the biosynthetic potential of these marine Streptomyces warrants further investigation, including the potential for biodiscovery of novel secondary metabolites from these isolates.

The highest intra-group sequence conservation, and therefore the lowest diversity, was seen within the Streptomyces albus group (Group B-SM9, SM17 and FMC008), suggesting that this group may prove to be less interesting in terms of potential discovery of novel bioactive secondary metabolites. While this hypothesis is supported by antiSMASH [29], analyses of the genomes of strains SM9 and FMC008 (which noticeably harbours the lowest abundance of smBGCs of any of the strains in this study-16 and 15 clusters, respectively) but not when considering the genome of strain SM17 (comprising 49 secondary metabolism gene clusters-the fourth highest abundance of any of the strains in this study).

\subsection{Secondary Metabolism Biosynthetic Gene Clusters}

The 13 Streptomyces spp. genomes were investigated for the presence of smBGCs of potential interest. All of the genomes examined contained numerous smBGCs as identified by antiSMASH (Table 3; Supplementary Tables S2-S14). The 13 strains contain a combined total of 485 individual clusters which share homology to 87 distinct known gene clusters whose metabolic product is known. While some clusters were common to many strains, all strains also contained clusters unique to that strain only (48 gene clusters are found in only one strain). The most commonly shared clusters were those showing similarities to the known clusters which produce the compatible solute ectoine (11 strains), the siderophore desferrioxamine B and the anti-tumour metabolite herboxidiene (7 strains), the peptide siderophore coelichelin, the carotenoid light-harvesting pigment isorenieratene and the terpene hopene (6 strains). The abundances or types of particular smBGCs are not a clear indicator of phylogenetic relatedness or of the isolation source (host sponge or sampling depth).

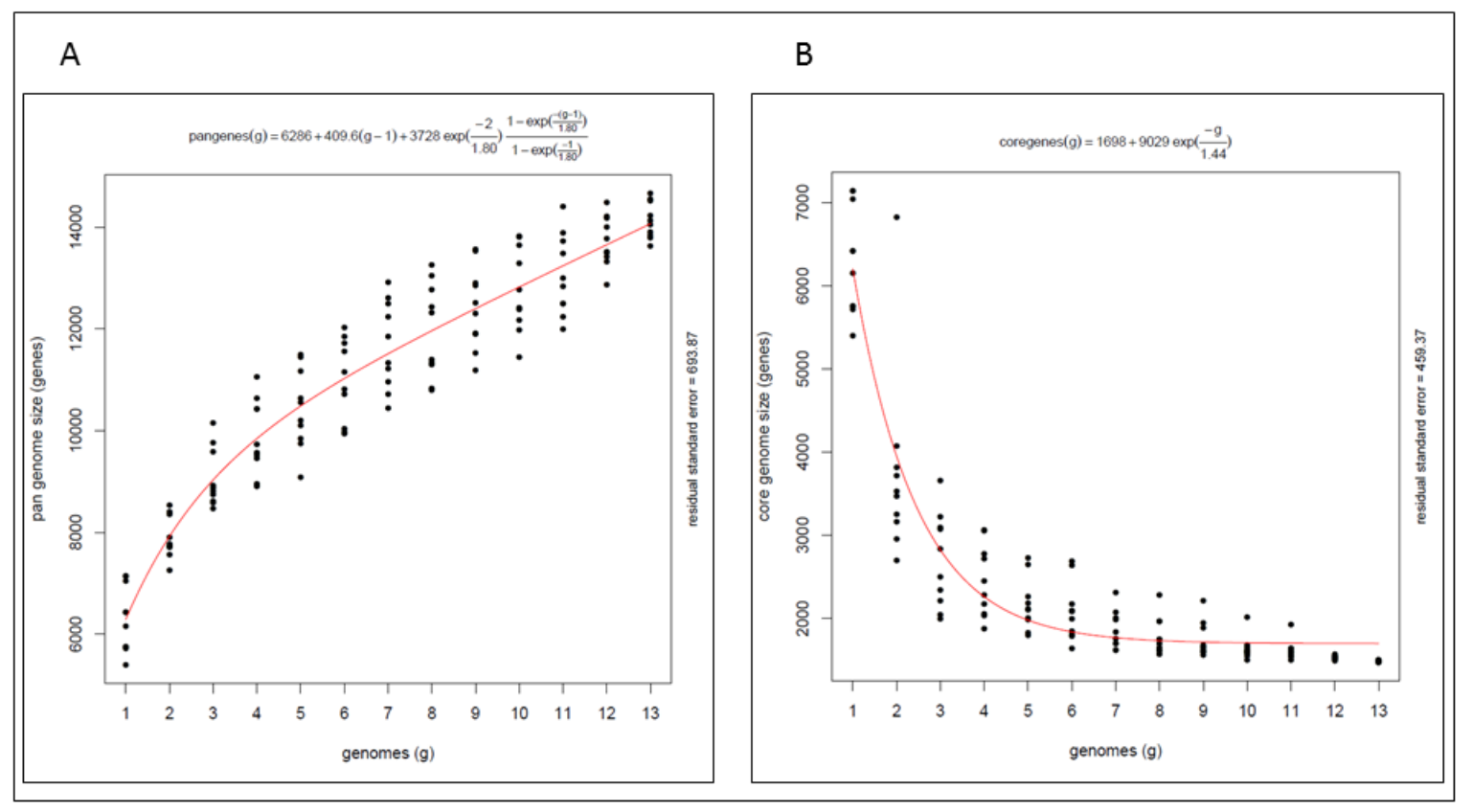

Figure 2. (A) Pangenome and (B) core genome of Streptomyces spp. marine isolates. 
Group A isolates display the largest genome sizes (Table 1) and with the exception of SM16 $(n=39)$ also possess the most abundant smBGCs $(n=51-54)$. The genomes of the Group A isolates show many similarities with all strains harbouring gene clusters related to the known clusters which produce the previously characterised metabolites ectoine, desferrioxamine B, coelichelin, herboxidiene and isorenieratene and also for $\gamma$-butyrolactone, alkylresorcinol and griseobactin. While these latter three metabolites are shared by all Group A strains, they are also exclusive to that group. Three of the four Group A strains also harbour gene clusters with similarities to the cluster known to produce the antibiotic friulimicin produced by the actinomycete Actinoplanes friuliensis, daptomycin, the heat-stable antifungal factor (HSAF) and also the peptide morphogen AmfS.

Gene clusters unique to one Group A isolate include those related to the production of thaxtomin, skyllamycin, balhimycin, griseoviridin, kanamycin, surfactin, myxothiazol A, pristinamycin, streptolydigin, concanamycin, chartreusin, naringenin, platensimycin, kosinostatin, oxazolomycin, bacillibactin, neocarzinostatin, glycolipopeptide, guadinomine and borrelidin.

Despite the phylogenetic relatedness of Group A isolates, the bioactivity profiles of the strains were not consistent. The deep-sea strains (B188M101, B226SN101) only displayed activity against yeasts while those sourced from shallow waters were only active against bacteria. Both Group A shallow water isolates (SM11, SM16) inhibited the growth of problematic drug resistant Staphylococcus spp. (Table 1). This makes further investigation of the smBGCs of these strains of high interest.

Groups B, C and D strains are less similar with few gene clusters shared by all members of any of those groups, except for ectoine biosynthesis genes which are highly similar in all Group B and Group C strains.

Two of the Group B strains (SM9, FMC008) harbour the fewest smBGCs (Table 3) in this study while the genome of the other Group B strain (SM17) is noticeably enriched in NRPS gene clusters. Analysis by antiSMASH shows that 15 of the 18 NRPS clusters in the SM17 genome show no similarities to known NRPS gene clusters. Analysis of Pfam domains of these clusters condensation (Supplementary Figures S1-S3) and epimerization domains (Supplementary Figure S5A) are diverse and abundant and form clades which correlate closely with phylogeny and show high similarities to those domains in the genome of S. albus J1074. All Group B strains harbour gene clusters with low similarity to the antimycin production cluster from S. albus J1074. Two of the three Group B strains also harbor clusters with low similarities to complestatin biosynthesis genes (SM9 and SM17) from S. albus J1074, the desotamide production cluster (SM9 and FMC008) from Myxococcus fulvus HW-1 and mannopeptimycin production genes (SM9 and FMC008) from Streptomyces hygroscopicus. The abundance of unknown NRPS gene clusters in the genome of SM17 as well as its broad range of antimicrobial activities versus E. coli and MRSA (Table 1) warrant further research efforts aimed at identifying the encoded metabolites.

Two of the three Group C strains contain gene clusters showing relatedness to the clusters known to produce mirubactin and paenibactin in Streptomyces sp. NTK 937, coelibactin in Streptomyces lividans TK24 and bafilomycin in Streptomyces lohii strain ATCC BAA-1276 (SM5 and SM18), together with cystothiazol A in Streptomyces griseus subsp. griseus NBRC 13350 and steffimycin in Streptomyces fulvissimus DSM 40593 (SM10 and SM18). This group contains numerous PKS, NRPS and PKS/NRPS hybrid clusters and display antibiotic activity against E. coli (SM5 and SM10) and against hVISA (SM10) and MRSA (SM18) while also inhibiting the growth of Bacillus spp. (Table 1). Thirteen of the smBGCs in the genome of SM18, including PKS, NRPS, PKS/NRPS hybrid, lantipeptide and bacteriocin clusters show no relatedness to known clusters in antiSMASH analysis. 
Table 3. Numbers of secondary metabolism gene clusters in the genomes of marine Streptomyces spp. isolates. * Numbers of PKS “types" (I, II \& III) can be seen in Supplementary Tables S2-S14.

\begin{tabular}{|c|c|c|c|c|c|c|c|c|c|c|c|}
\hline Isolate ID & PKS * & NRPS & PKS/NRPS Hybrid & Bacteriocin & Lantipeptide & Siderophore & Terpene & Butyrolactone & Ectoine & Other & TOTAL \\
\hline B188M101 & 4 & 19 & 4 & 2 & 4 & 2 & 5 & 2 & 2 & 9 & 53 \\
\hline B226SN101 & 2 & 20 & 4 & 2 & 3 & 2 & 5 & 2 & 2 & 9 & 51 \\
\hline SM5 & 5 & 9 & 5 & 2 & 1 & 1 & 6 & 1 & 1 & 7 & 38 \\
\hline SM10 & 16 & 3 & 3 & 5 & 3 & 2 & 4 & 2 & 1 & 5 & 44 \\
\hline SM11 & 10 & 11 & 5 & 2 & 3 & 2 & 6 & 2 & 2 & 11 & 54 \\
\hline SM12 & 18 & 7 & - & 1 & 3 & 4 & 1 & 1 & 1 & 4 & 40 \\
\hline SM16 & 2 & 5 & 6 & 1 & 5 & 2 & 7 & 2 & 2 & 7 & 39 \\
\hline SM1 & 4 & 8 & 1 & 2 & 2 & 2 & 4 & 1 & - & 5 & 28 \\
\hline SM9 & 3 & 2 & - & 1 & - & 2 & 6 & - & 1 & 1 & 16 \\
\hline FMC008 & 1 & 3 & - & 1 & - & 1 & 5 & - & 1 & 3 & 15 \\
\hline SM18 & 9 & 9 & 6 & 2 & 2 & 1 & 6 & 1 & 1 & 4 & 41 \\
\hline SM14 & 8 & 2 & - & - & 1 & 2 & - & 1 & - & 3 & 17 \\
\hline SM17 & 14 & 18 & 2 & 3 & 2 & 3 & 5 & - & 1 & 1 & 49 \\
\hline
\end{tabular}


The only secondary metabolism gene cluster common to Group D isolates displays low levels of similarity to the cluster which produces zorbamycin in Streptomyces flavoviridis ATCC 21892. Both Group D strains (SM12 and SM14) inhibited the growth of hVISA (Table 1) making them of particular interest because they appear phylogenetically distant from all other strains presented here. The strains could not be assigned to a genus in Kraken analysis (Supplementary Table S1) and appear phylogenetically distant in FFP analysis (Figure 1B). The genome of SM12 hosts 18 Type I PKS clusters of which 10 share no homology with known gene clusters in the linked Minimum Information about a Biosynthetic Gene Cluster (MIBiG) database (https: / / mibig.secondarymetabolites. org/index.html) [30]. Increasing the taxonomic diversity of available Streptomyces spp. strains available for screening regimes is predicted to increase the chemical diversity of identified metabolites. With that in mind, these phylogenetically remote strains with demonstrated bioactivities and numerous uncharacterised PKS gene cluster products are of high interest.

The genome of SM1, which does not recruit to the groups (A-D) described here, also harbours a unique secondary metabolism gene cluster profile. While SM1 does harbour clusters for the frequently observed desferrioxamine B and isorenieratene production genes, it is one of only two genomes presented here not to encode ectoine biosynthesis genes. Of the 28 gene clusters in the genome of SM1, as identified by antiSMASH, only nine of those clusters show homology to known gene clusters and three of these are unique to SM1 in our study. As this strain has displayed broad-range antimicrobial activity and appears only distantly related to other strains in Figure $1 \mathrm{~B}$ and distantly related to the metabolically talented S. coelicolor A3(2) in Kraken analysis (Supplementary Table S1), it also warrants further investigation with respect to the further characterization of its metabolites.

\subsection{Protein Family (Pfam) Domain Analysis}

The genome assemblies of the 13 marine Streptomyces strains were highly fragmented, and so smBGC prediction can be hampered by particular clusters being fragmented across different contigs. This can lead to an over-prediction of the numbers of clusters present. Nonetheless it was possible to analyse deduced proteins at the domain level. Translated sequences of protein domains of interest: ketosynthase (KS) domains of PKS genes (Supplementary Figure S1), condensation starter domains (Supplementary Figure S2), condensation DCL domains (Supplementary Figure S3), condensation LCL domains (Supplementary Figure S4) epimerization (Supplementary Figure S5A) and heterocyclization (Supplementary Figure S5B) domains of NRPS gene clusters, IucA-IucC domains of siderophore genes (Supplementary Figure S6), DUF692 domains of bacteriocin genes (Supplementary Figure S7) and LanC domains of lantibiotic genes (Supplementary Figure S8) were extracted from the antiSMASH results using a custom script. These domains were analysed and are presented as a heat-map (Figure 3) with the evolutionary relationships of these protein domains shown in phylogenetic trees (Supplementary Figures S1-S8). 


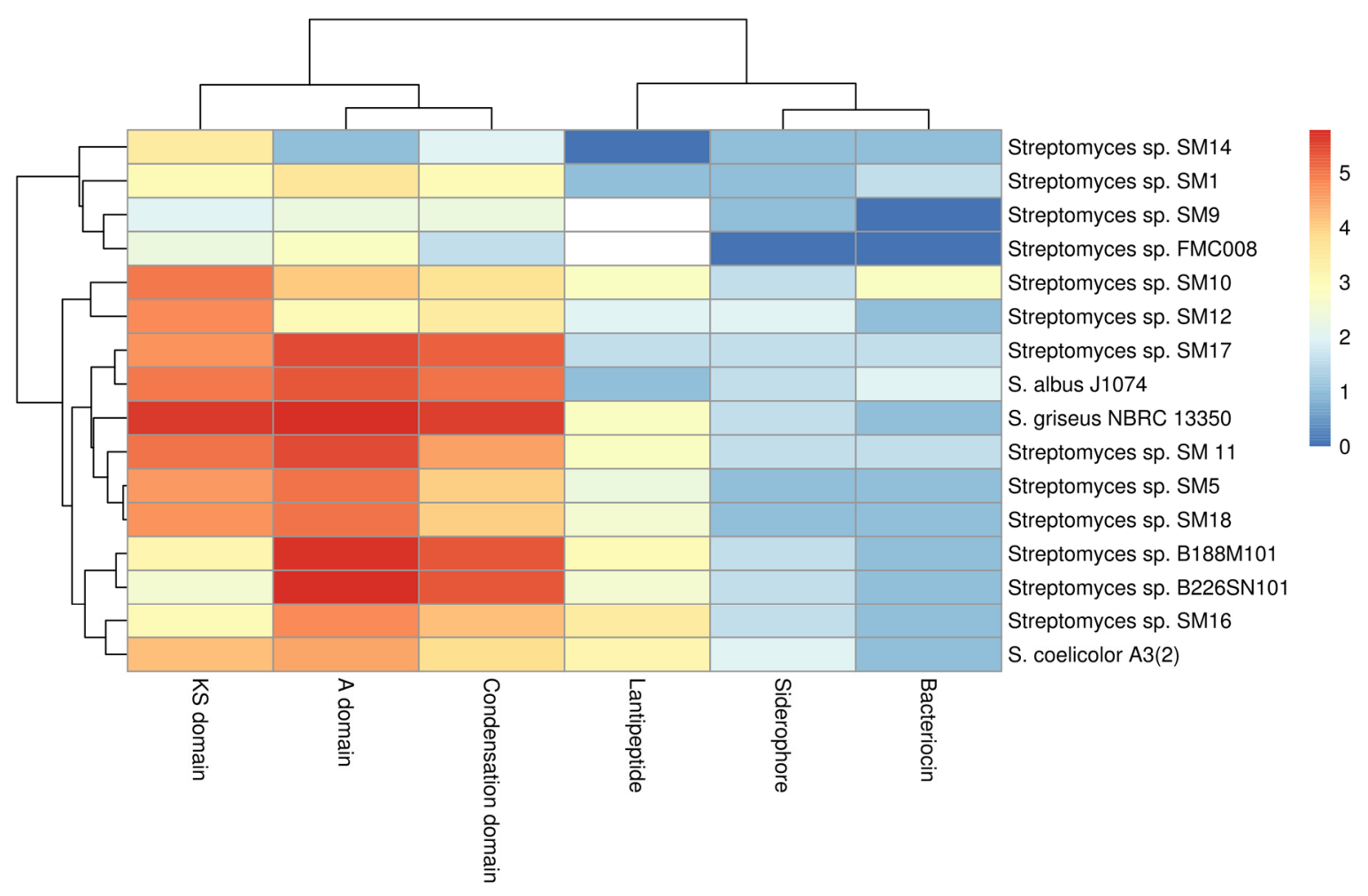

Figure 3. $\log 2$ heatmap of predicted secondary metabolism protein domains of interest from marine Streptomyces spp. isolates and from select reference terrestrial genomes.

\subsubsection{KS Domains of PKS Gene Clusters}

Of 25 KS domain sub-clades identified (Supplementary Figure S1), 12 of these clades include only one of the phylogenetic sub-groups (A-D) described earlier (Figure 1B), suggesting a link between the phylogeny of the isolates and a subset of the KS domains therein. As the remaining 13 clades of KS domains contain domains from two or more phylogenetic subgroups, it appears that these KS domains are not phylogeny-related but represent a more general genetic diversity. It is clear from the tree (Supplementary Figure S1) that the number of clades and the individual branch lengths, indicate that very high levels of diversity are apparent even within a single protein domain type amongst a limited number of isolates from this single genus.

\subsubsection{Nrps Gene Clusters}

Protein domains from NRPS clusters (condensation starter, condensation DCL, condensation LCL, epimerization and heterocyclization were analysed individually by sequence alignments and phylogenetic tree building (Supplementary Figures S2-S5). Thirteen distinct clades of condensation starter domains were observed (Supplementary Figure S2). These domains appear diverse but are more closely distinguishable as recruiting to phylogenetic groups A-D (Figure 1B) than is observed in the KS domain analysis (Supplementary Figure S1). Six of the clades contain domains from only one phylogenetic sub-group while one of these clades includes domains from all four phylogenetic sub-groups. It is noted that a single isolate may contain more than one condensation starter domain gene (where more than one NRPS gene cluster is present). These condensation starter domains may be highly similar (e.g., condensation starter domains of SM9- Supplementary Figure S2) or indeed very different (e.g., condensation starter domains of SM12-Figure S2). It is also noteworthy that although the genome of SM14 harbours two NRPS gene clusters, no condensation starter domain has been identified in these clusters. When considering other NRPS domains, condensation DCL (Supplementary Figure S3) and condensation LCL (Supplementary Figure S4), 
epimerization (Supplementary Figure S5A) and heterocyclization (Supplementary Figure S5B) domains, many phylogenetic clades harbour sequences which correlate with the taxonomic phylogeny described in Figure 1B. As before, no epimerization or heterocyclization domains were identified in the NRPS clusters of Group D strains (SM12 and SM14). Nonetheless, even within the clades identified, noticeable degrees of diversity are apparent (i) within strains with multiple gene clusters, (ii) within phylogenetic clades and (iii) overall. The tree topologies and branch lengths indicate high levels of gene diversity in marine Streptomyces spp. Group A isolates (SM11, SM16, B188M101 and B226SN101) appear enriched for adenylation (Figure 3) and condensation (Supplementary Figures S2-S4) domains of NRPS gene clusters when compared to the other phylogenetic sub-groups described here. There appears to be a stronger link between taxonomic phylogeny and the evolutionary phylogeny of NRPS epimerization (Supplementary Figure S5A) and NRPS heterocyclization domains (Supplementary Figure S5B). These domains are highly abundant in Group A and Group B isolates but entirely absent from the genomes of Group D strains. The majority of predicted protein domains from the deep sea isolates (B188M101 and B226SN101) are more similar to each other than to similar genes in shallow water or terrestrial isolates. This supports previous findings by our group where we reported putative deep sea sponge specific microbiome [24] and secondary metabolome [31].

\subsubsection{Siderophores, Bacteriocins and Lantibiotics}

Phylogeny-related patterns of predicted protein domains of siderophores (IucA-IucCSupplementary Figure S6), bacteriocins (DUF692—Supplementary Figure S7) and lantipeptides (LanC-like-Supplementary Figure S8) were notable with the majority of those domains identified here, clustering more closely to domains from within the phylogenetic subgroups than to those of other subgroups. Bioavailable iron in ocean waters has long been recognized as a limiting factor for growth [32]. Dissolved iron concentrations are orders of magnitude higher in surface and mesopelagic waters when compared to the deep sea [33]. Thus, it might be expected that microbes from deep waters may contain more genes involved in iron chelation or with higher degrees of siderophore gene diversity. We have not observed this however and possible explanations may be that the genes in the deep sea strains are more highly transcribed than those of strains from shallower waters, or that the symbiotic relationship between sponges and their resident microbiota may provide higher concentrations of iron to the microbes than is available to planktonic ocean microorganisms. Iron has been identified as an important mineral for sponge primmorph proliferation and morphogenesis [34] and it has been demonstrated that some sponges can accumulate trace elements, including iron, at concentrations high above those of seawater and sediments [35].

Eleven of the 13 genomes described here host DUF692 domains found in bacteriocin production genes, the exceptions being SM9 and FMC008 despite antiSMASH predictions of one bacteriocin gene cluster in each of those genomes. Conversely, the genome of SM14 hosts a DUF692 domain, but no bacteriocin cluster was predicted for this strain by antiSMASH. Although five NRPS gene clusters were predicted in the genome of SM10 by antiSMASH, only three DUF692 domains were identified. Nonetheless, two of those domains are notably different to all other DUF692 domains identified here and to each other. The genomes of all other strains host one DUF692 gene copy each. Degrees of conservation in all deduced protein sequences apart from the aforementioned SM10 sequences are evident from the phylogenetic tree (Supplementary Figure S7), though an evolutionary pattern correlated with taxonomic phylogeny can also be seen.

When considering the lantibiotic (LanC-like) domains, Groups A (SM11, SM16, B188M101 and B226SN101) and C (SM5, SM10 and SM18) as well as the terrestrial strains S. griseus and S. coelicolor, hosted more potential LanC-like domains when compared to Groups B (SM9, SM17 and FMC008) and D (SM12 and SM14) (Supplementary Figure S8). Strains B188M101 and SM16 hosted the most lantibiotic smBGCs in this study (four and five respectively) and also hosted the most LanC-like protein domain gene sequences (five and seven respectively). All seven of these protein domains on 
the SM16 genome are quite different to each other indicating that diverse lantipeptides may potentially be produced by that strain.

\subsection{Marine Adaptations}

The 13 marine Streptomyces genomes were interrogated for the presence of genes that may be potentially involved in the biosynthesis and/or transport of compatible solutes and other osmoregulatory systems, which have previously been suggested to play a role in marine adaptation of microorganisms [36]. The abundances of these genes were compared to abundances in the terrestrial Streptomyces spp. genomes which were used to construct the Ffp phylogenetic tree (Figure 4).

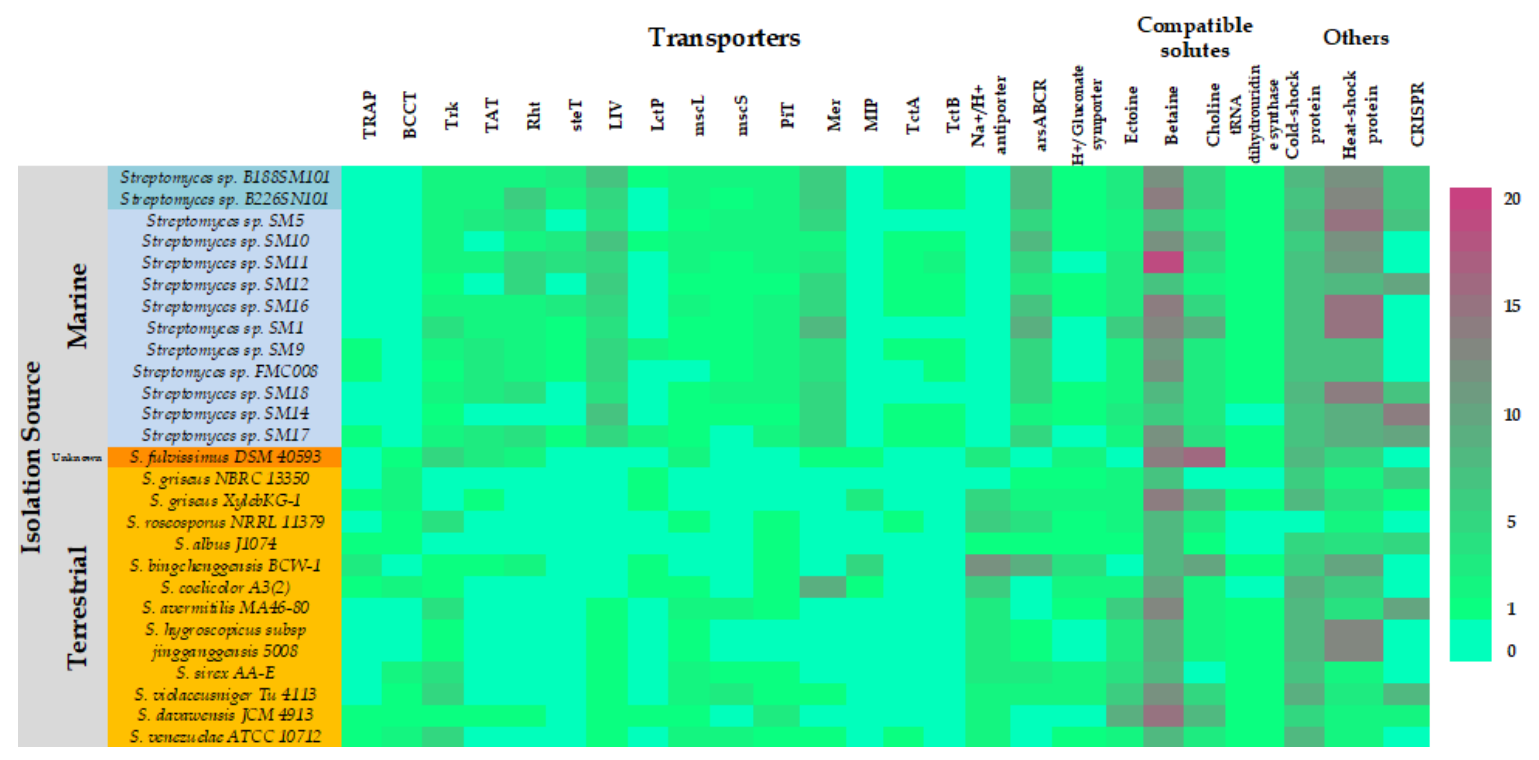

Figure 4. Marine adaptations in Streptomyces spp.: Abundances of compatible solutes and osmoprotectant biosynthesis and/or transport genes and of CRISPR genes in the genomes of marine Streptomyces spp. isolates (blue background) and reference terrestrial Streptomyces spp. genomes (orange background). TRAP: Tripartite ATP-dependent Periplasmic transporters (organic acid import); BCCT: Betaine Carnitine Choline Transporter; Trk: Potassium ion transport; TAT: Twin Arginine Translocator; Rht: homoserine/threonine transport; steT: serine/ threonine exchanger transporter; LIV: Transport of Branched-Chain Amino Acids; LctP: lactose permease; mscL: Large Conductance Mechanosensitive Ion Channel; mscS: Small Conductance Mechanosensitive Ion Channel; PiT: Sodium-dependent phosphate transporter; Mer: Mercury transporter; MIP: major intrinsic protein; TctA: Tripartite tricarboxylate transporter; TctB: Tripartite tricarboxylate transporter; arsABCR: Arsenite detoxification system.

The marine isolates appear to be enriched for mercury and arsenic transport systems, branched-chain amino acid transport (LIV), betaine and choline biosynthesis and heat shock proteins. The marine strains appeared however to be deficient in sodium-proton antiporters, tripartite ATP independent periplasmic transporters (TRAP), betaine transport (BCCT), serine/threonine export (Rht), aquaporins (MIP) transport and cold shock proteins. It is interesting that the genomes of the marine strains are enriched for the arsenic transport genes in light of the recent finding that sponge associated Entotheonella spp. sequester arsenic in intracellular vesicles whilst residing in its sponge host, Theonella swinhoei [37]. More work will be necessary to determine if the Streptomyces strains described here are preferentially exporting arsenic or importing and sequestering it as a host-protection symbiotic function. Our analysis also suggests that betaine may be the preferred compatible solute amongst marine Streptomyces spp. as indicated by the higher abundances of betaine biosynthetic genes when compared to those of ectoine or choline. This however needs to be experimentally confirmed by assessing transcription rates of those genes. 


\section{Materials and Methods}

\subsection{Sponge Sampling}

The marine sponge Haliclona simulans (class Demospongiae, order Haplosclerida, family Chalinidae) was sampled by SCUBA diving at a depth of $15 \mathrm{~m}$ in Kilkieran Bay, Galway, Ireland (N 53 $18^{\prime} 56.6^{\prime \prime}$, W $09^{\circ} 40^{\prime} 08.4^{\prime \prime}$ ) [24]. The sponge Lissodendoryx diversichela (class Demospongiae, order Poecilosclerida, family Coelosphaeridae) was sampled using the ROV Holland I from the RV Celtic Explorer at a depth of $1350 \mathrm{~m}$ in the North Atlantic Ocean (N 54 $21^{\prime} 25.2^{\prime \prime}$, W $\left.12^{\circ} 14^{\prime} 49.9^{\prime \prime}\right)$ off the west coast of Ireland [25]. The sponge Inflatella pellicula (class Demospongiae; order Poecilosclerida; suborder Myxillina; family Coelosphaeridae) was sampled using the ROV Holland I at a depth of $2900 \mathrm{~m}$ in the North Atlantic Ocean (N $54^{\circ} 03^{\prime} 28.8^{\prime \prime}, \mathrm{W} 12^{\circ} 32^{\prime} 53.5^{\prime \prime}$ ) [26].

\subsection{Culture Isolation}

Sponge samples were rinsed with sterile artificial seawater (3.33\% Instant Ocean ${ }^{\mathrm{TM}}$ - Aquatic Eco-Systems Inc., Apopka, FL, USA) to remove exogenous materials. Sponge tissues ( $1 \mathrm{~g})$ were macerated with a sterile razor blade. Serial dilutions of the macerated tissues were spread to 3 isolation media; (i) starch-yeast-peptone seawater agar (SYP-SW): $1 \%(w / v)$ starch, $0.4 \%(w / v)$ yeast extract, $0.2 \%(w / v)$ peptone, 3.33\% $(w / v)$ artificial sea salts (Instant Ocean $\left.{ }^{\mathrm{TM}}\right), 1.5 \%(w / v)$ agar; (ii) modified marine agar (MMA): $0.005 \%(w / v)$ yeast extract, $0.05 \%(w / v)$ tryptone, $0.01 \%(w / v)$ $\beta$-glycerol phosphate disodium salt, pentahydrate, 3.33\% $(w / v)$ artificial sea salts (Instant Ocean ${ }^{\mathrm{TM}}$, Apopka, FL, USA), 1.5\% (w/v) agar and (iii) chitin agar: $4 \%(v / v)$ colloidal chitin, $1.5 \%(w / v)$ agar. Culture plates were incubated at $28^{\circ} \mathrm{C}$ for up to 8 weeks. Colonies were picked from isolation plates, streaked to fresh agar plates to obtain pure, isolated colonies. Isolates were archived by growing in liquid broth (SYP-SW) supplemented with $15 \%(v / v)$ glycerol and frozen at $-80{ }^{\circ} \mathrm{C}$.

\subsection{DNA Extraction}

Isolates were grown overnight $(\sim 16 \mathrm{~h})$ in $5 \mathrm{~mL}$ liquid cultures. Cells were pelleted by centrifugation $(6000 \mathrm{~g})$, supernatants were decanted and discarded. Cell pellets were resuspended in $467 \mu \mathrm{L} \mathrm{TE}$ buffer. $30 \mu \mathrm{L}$ of $10 \%$ SDS and $3 \mu \mathrm{L}$ of $20 \mathrm{mg} / \mathrm{mL}$ Proteinase K (Fermentas, Sankt Leon-Rot, Germany) was added to each tube and incubated for $1 \mathrm{~h}$ at $37^{\circ} \mathrm{C}$. An equal volume of phenol/chloroform (phenol-chloroform-isoamyl alcohol mixture ratio 25:24:1, Sigma Aldrich, Arklow, Ireland) was added and mixed well. Tubes were centrifuged, at $\sim 18,042 \mathrm{~g}$ for $10 \mathrm{~min}$ (Eppendorf Centrifuge, 5417r, Eppendorf UK Ltd., Stevenage, UK). The upper phase of each Eppendorf tube was aspirated to fresh $2 \mathrm{~mL}$ Eppendorf tubes, avoiding the interphase. $100 \mu \mathrm{L}$ of $3 \mathrm{M}$ sodium acetate (NaOAc) pH 5.2 was added to each tube and mixed well. $600 \mu \mathrm{L}$ of isopropanol (Sigma Aldrich, Arklow, Ireland) was added, mixed well, and incubated at room temperature for $15 \mathrm{~min}$. Tubes were then centrifuged at $\sim 18,042 \mathrm{~g}$ for $20 \mathrm{~min}$. Supernatants were removed and discarded. DNA pellets were washed with cold $\left(4{ }^{\circ} \mathrm{C}\right) 70 \% \mathrm{EtOH}$. Tubes were centrifuged at $\sim 18,042 \mathrm{~g}$ for $10 \mathrm{~min}$. Ethanol was removed and discarded and DNA pellets were allowed to air-dry. DNA was re-suspended in $1 \mathrm{~mL} \mathrm{TE} \mathrm{buffer.} 1 \mu \mathrm{L}$ of RNase A was added to the tubes, which were then incubated at $37^{\circ} \mathrm{C}$ for $30 \mathrm{~min}$. DNA was again purified by phenol extraction. DNA was analysed by gel electrophoresis and quantified using a spectrophotometer (NanoDrop ND-1000, Thermo Scientific, Gloucester, UK). The DNA solutions were stored at $-20^{\circ} \mathrm{C}$.

\section{4. $16 \mathrm{~S}$ rRNA Gene Sequencing}

16S rRNA genes were amplified by PCR using a Peltier Thermal Cycler PTC-200 PCR System (MJ Research Inc., Waltham, MA, USA). Each PCR reaction comprised 1X reaction buffer, $0.2 \mathrm{mM}$ dNTPs, $0.5 \mu \mathrm{M}$ forward primer [27f ( $5^{\prime}$-AGAGTTTGATCMTGGCTCAG-3')], $0.5 \mu \mathrm{M}$ reverse primer [1492r (5'-TACGGYTACCTTGTTACGACTT - $\left.3^{\prime}\right)$ ] [38], 1 U Taq polymerase $(5 \mathrm{U} / \mu \mathrm{L}), 1.0 \mu \mathrm{L}$ template DNA (1-10 ng), sdH2O. PCR cycle conditions comprised initial denaturation at $95{ }^{\circ} \mathrm{C}$ for $3 \mathrm{~min}$ followed by 30 cycles of denaturation at $95^{\circ} \mathrm{C}$ for $1 \mathrm{~min}$, primer annealing at $50^{\circ} \mathrm{C}$ for $1 \mathrm{~min}$, extension at $72{ }^{\circ} \mathrm{C}$ for 
$1 \mathrm{~min}$ followed by a final extension at $72{ }^{\circ} \mathrm{C}$ for $5 \mathrm{~min}$. PCR products were analysed by electrophoresis on $1 \%$ agarose gels. PCR amplicons were sequenced by capillary electrophoresis, single extension sequencing (Macrogen Inc., Korea), using 3730xl DNA Analyser.

\section{5. $16 \mathrm{~S}$ rRNA Based Phylogenetic Analysis}

Sequences were manually edited for quality using FinchTV 1.4.0 (Geospiza, Inc.; Seattle, WA, USA; http:/ / www.geospiza.com). Sequence alignment and tree construction were performed using MEGA version 6 [39] (Arizona State University \& Pennsylvania State University, USA, http:/ / www. megasoftware.net/). Alignment was performed with ClustalW [40] using the Maximum Parsimony approach [41] and included bootstrap tests $(n=500)$. The evolutionary distances are in the units of the number of base substitutions per site. All positions containing gaps and missing data were eliminated from the datasets (complete deletion option). Reference sequences were downloaded from the Ribosomal Database Project (release 11, update 4, 26 May 2015, https:/ /rdp.cme.msu.edu/) [42].

\subsection{Bioactivity Screening}

Isolates were screened for antibacterial activities against a panel of clinically relevant Gram negative [Escherichia coli NCIMB 12210 and Pseudomonas aeruginosa PAO1], Gram positive [Bacillus spp.-B. subtilis 1E32, B. subtilis 1A40, B. cereus FPL1); Staphylococcus spp. (hVISA [Heterogonous Vancomycin Intermediate Staphylococcus aureus 22900], MRSA [Methicillin Resistant S. aureus ST544], VISA [Vancomycin Intermediate S. aureus 35403], S. aureus NCIMB 9518) and Listeria monocytogenes F2365] and against the yeasts Candida spp. [C. glabrata CBS138 and C. albicans MY1055] and the fungus Aspergillus fumigatus ATCC 46645 test strains using a deferred antagonism assay [43]. It should be noted that not all isolates were tested against all test strains. Only the results shown in Table 1 were performed. Isolates were spotted to the centre of Petri dishes on SYP-SW agar and grown until the colony reached $1-2 \mathrm{~cm}$ in diameter. Test strains were grown overnight in $5 \mathrm{~mL}$ LB broth, shaking, $200 \mathrm{rpm}$ until they reached an $0 \mathrm{D} 600_{\mathrm{nm}} 0.8$. Test strains were then diluted 1:50 in LB soft agar $(0.7 \%$ agar- $w / v)$. Inoculated soft agar was poured onto the surface of the plates containing the isolates. Plates were incubated at $37^{\circ} \mathrm{C}$ and examined the next day for zones of inhibition-clearance zones in the growth of the test strain around the colony of the isolate.

\subsection{Whole Genome Sequencing}

For whole genome sequencing, mate pair libraries were prepared the using the Nextera XT DNA Library Preparation Kit (Illumina, San Diego, CA, USA) according to the manufacturer's instructions. All libraries were sequenced in 250 bp paired read runs on the Illumina MiSeq platform. Reads were trimmed for quality with Sickle (Available at https: / / github.com/najoshi/sickle) and Scythe (Available at https://github.com/vsbuffalo/scythe).

\subsection{Bioinformatic Methods}

Genome assemblies were performed using SPAdes, version 3.1.1 using K-mer lengths of 21, 33, 55, 77, 81 and 91 [44]. Pan genome analysis was performed using Get Homologues [45]. Comparative genomic methods were carried out on the following 25 genomes which included the 13 isolates from Irish marine sponges presented here and the following 12 terrestrial Streptomyces spp. genomes from the sequence databases: S. albus J1074, S. avermitilis MA46-80, S. bingchenggensis BCW-1, S. coelicolor A3(2), S. davaoensis JCM 4913, S. fulvissimus DSM 40593, S. hygroscopicus subsp jingganggensis 5008, S. griseus NBRC 13350, S. lividans, S. sirex AA-E, S. venezuelae ATCC 10712 and S. violaceusniger Tü4113. Potential bioactive gene clusters were identified by searching the genomes with antiSMASH version 3 and parsed with $\mathrm{R}$ methods, $\mathrm{R}$ is a free software environment for statistical computing and graphics (The R Project-available at https:/ / www.r-project.org/). Pfam domains often represented within these gene clusters were counted and graphed using $\mathrm{R}$. 


\section{Conclusions}

Analysis of the draft genomes of 11 Streptomyces spp. isolated from a shallow water sponge and an additional two isolated from deep sea sponges reveal the presence of highly abundant smBGCs. Amongst the 485 gene clusters identified, the majority display little or no homology with known smBGCs in the MIBiG database. The notably abundant PKS, NRPS, PKS/NRPS hybrid, bacteriocin and lantipeptide gene clusters are of particular interest in the search for novel antibiotic families and species in light of the worrisome trends in the occurrences of antimicrobial resistant human pathogens, particularly so because many of these strains have effectively inhibited the growth of problematic pathogenic strains (hVISA, MRSA) in plate screens.

Availability of data and material: The datasets generated during and/or analysed during the current study are available in GenBank hosted by NCBI under the BioProject accession PRJNA384120 (https:/ / www.ncbi.nlm.nih.gov/bioproject/PRJNA384120/) with individual genome accession codes NETW00000000, NETX00000000, NETY00000000, NETZ00000000, NEUA00000000, NEUB00000000, NEUC00000000, NEUD00000000, NEUE00000000, NEUF00000000, NEUG00000000, NEUH00000000, NEUI00000000.

Supplementary Materials: The following are available online at www.mdpi.com/1660-3397/16/2/67/s1, Figure S1: Phylogenetic tree of deduced amino acid sequences of KS domains from PKS gene clusters from Streptomyces spp.; Figure S2: Phylogenetic tree of deduced amino acid sequences of condensation starter domains of NRPS gene clusters from Streptomyces spp.; Figure S3: Phylogenetic tree of deduced amino acid sequences of condensation DCL domains NRPS gene clusters from Streptomyces spp.; Figure S4: Phylogenetic tree of deduced amino acid sequences of condensation LCL domains from NRPS gene clusters from Streptomyces spp.; Figure S5: Phylogenetic tree of deduced amino acid sequences of (A) epimerization domains and (B) heterocyclization domains from NRPS gene clusters from Streptomyces spp.; Figure S6: Phylogenetic tree of deduced amino acid sequences of IucA-IucC domains from PKS gene clusters from Streptomyces spp.; Figure S7: Phylogenetic tree of deduced amino acid sequences of DUF692 domains from bacteriocin gene clusters from Streptomyces spp.; Figure S8: Phylogenetic tree of deduced amino acid sequences of LanC-like domains from lantipeptide gene clusters from Streptomyces spp.; Table S1: Taxonomy of Streptomyces spp. isolates using KRAKEN; Table S2: Secondary metabolism biosynthetic gene clusters in the genome of Streptomyces sp. B188M101 as predicted by antiSMASH; Table S3: Secondary metabolism biosynthetic gene clusters in the genome of Streptomyces sp. B226SN101 as predicted by antiSMASH; Table S4: Secondary metabolism biosynthetic gene clusters in the genome of Streptomyces sp. SM5 as predicted by antiSMASH; Table S5: Secondary metabolism biosynthetic gene clusters in the genome of Streptomyces sp. SM10 as predicted by antiSMASH; Table S6: Secondary metabolism biosynthetic gene clusters in the genome of Streptomyces sp. SM11 as predicted by antiSMASH; Table S7: Secondary metabolism biosynthetic gene clusters in the genome of Streptomyces sp. SM12 as predicted by antiSMASH; Table S8: Secondary metabolism biosynthetic gene clusters in the genome of Streptomyces sp. SM16 as predicted by antiSMASH; Table S9: Secondary metabolism biosynthetic gene clusters in the genome of Streptomyces sp. SM17 as predicted by antiSMASH; Table S10: Secondary metabolism biosynthetic gene clusters in the genome of Streptomyces sp. SM18 as predicted by antiSMASH; Table S11: Secondary metabolism biosynthetic gene clusters in the genome of Streptomyces sp. SM1 as predicted by antiSMASH; Table S12: Secondary metabolism biosynthetic gene clusters in the genome of Streptomyces sp. FMC008 as predicted by antiSMASH; Table S13: Secondary metabolism biosynthetic gene clusters in the genome of Streptomyces sp. SM14 as predicted by antiSMASH; Table S14: Secondary metabolism biosynthetic gene clusters in the genome of Streptomyces sp. SM9 as predicted by antiSMASH.

Acknowledgments: This study was funded by the EU FP7 PharmaSea Project (312184 PHARMASEA) and by the Earth and Life Systems Alliance (ELSA), Norwich Research Park. DNA sequencing was funded by the Earth and Life Systems Alliance (ELSA), Norwich Research Park.

Author Contributions: A.D.W.D. conceived and designed the study. Strain isolation, bioactivity screening and DNA preparation was performed by S.A.J., L.M.M. and J.K. Sequencing and genome assemblies were performed by L.C. Bioinformatic analyses were performed by S.A.J., L.C., E.L.A. and L.M.M. Manuscript was written by S.A.J., L.C. and A.D.W.D.

Conflicts of Interest: The authors declare that they have no competing interests. 


\section{References}

1. Subramani, R.; Aalbersberg, W. Culturable rare actinomycetes: Diversity, isolation and marine natural product discovery. Appl. Microbiol. Biotechnol. 2013, 97, 9291-9321. [CrossRef] [PubMed]

2. Berdy, J. Bioactive microbial metabolites. J. Antibiot. (Tokyo) 2005, 58, 1-26. [CrossRef] [PubMed]

3. Tommasi, R.; Brown, D.G.; Walkup, G.K.; Manchester, J.I.; Miller, A.A. ESKAPEing the labyrinth of antibacterial discovery. Nat. Rev. Drug Discov. 2015, 14, 529-542. [CrossRef] [PubMed]

4. Wright, G.D. Antibiotics: A New Hope. Chem. Biol. 2012, 19, 3-10. [CrossRef] [PubMed]

5. Lewis, K. Platforms for antibiotic discovery. Nat. Rev. Drug Discov. 2013, 12, 371-387. [CrossRef] [PubMed]

6. Craig, J.W.; Chang, F.Y.; Kim, J.H.; Obiajulu, S.C.; Brady, S.F. Expanding small-molecule functional metagenomics through parallel screening of broad-host-range cosmid environmental DNA libraries in diverse proteobacteria. Appl. Environ. Microbiol. 2010, 767, 1633-1641. [CrossRef] [PubMed]

7. Ekkers, D.M.; Creitoiu, M.S.; Kielak, A.M.; Elsas, J.D. The great sreen anomaly-A new frontier in product discovery through functional metagenomics. Appl. Microbiol. Bitechnol. 2012, 93, 1005-1020. [CrossRef] [PubMed]

8. Seyedsayamdost, M.R. High-throughput platform for the discovery of elicitors of silent bacterial gene clusters. Proc. Natl. Acad. Sci. USA 2014, 111, 7266-7271. [CrossRef] [PubMed]

9. Wu, Y.; Seyedsayamdost, M.R. Synergy and Target Promiscuity Drive Structural Divergence in Bacterial Alkylquinolone Biosynthesis. Cell Chem. Biol. 2017, 24, 1437-1444.e3. [CrossRef] [PubMed]

10. Smanski, M.J.; Zhou, H.; Claesen, J.; Shen, B.; Fischbach, M.A.; Voigt, C.A. Synthetic biology to access and expand nature's chemical diversity. Nat. Rev. Microbiol. 2016. [CrossRef] [PubMed]

11. Reen, F.J.; Romano, S.; Dobson, A.D.; O'Gara, F. The Sound of Silence: Activating Silent Biosynthetic Gene Clusters in Marine Microorganisms. Mar. Drugs 2015, 13, 4754-4783. [CrossRef] [PubMed]

12. Rosen, P.C.; Seyedsayamdost, M.R. Though Much Is Taken, Much Abides: Finding New Antibiotics Using Old Ones. Biochemistry 2017, 56, 4925-4926. [CrossRef] [PubMed]

13. Xu, F.; Nazari, B.; Moon, K.; Bushin, L.B.; Seyedsayamdost, M.R. Discovery of a Cryptic Antifungal Compound from Streptomyces albus J1074 Using High-Throughput Elicitor Screens. J. Am. Chem. Soc. 2017, 139, 9203-9212. [CrossRef] [PubMed]

14. Gomez-Escribano, J.; Alt, S.; Bibb, M. Next Generation Sequencing of Actinobacteria for the Discovery of Novel Natural Products. Mar. Drugs 2016, 14, 78. [CrossRef] [PubMed]

15. Jensen, P.R.; Moore, B.S.; Fenical, W. The marine actinomycete genus Salinispora: A model organism for secondary metabolite discovery. Nat. Prod. Rep. 2015, 32, 738-751. [CrossRef] [PubMed]

16. Alt, S.; Wilkinson, B. Biosynthesis of the Novel Macrolide Antibiotic Anthracimycin. ACS Chem. Biol. 2015, 10, 2468-2479. [CrossRef] [PubMed]

17. Moon, K.; Ahn, C.H.; Shin, Y.; Won, T.H.; Ko, K.; Lee, S.K.; Oh, K.B.; Shin, J.; Nam, S.I.; Oh, D.C. New benzoxazine secondary metabolites from an arctic actinomycete. Mar. Drugs 2014, 12, 2526-5238. [CrossRef] [PubMed]

18. Yuan, M.; Yu, Y.; Li, H.R.; Dong, N.; Zhang, X.H. Phylogenetic diversity and biological activity of actinobacteria isolated from the Chukchi shelf marine sediments in the Arctic Ocean. Mar. Drugs 2014, 12, 1281-1297. [CrossRef] [PubMed]

19. Kamjam, M.; Sivalingam, P.; Deng, Z.; Hong, K. Deep Sea Actinomycetes and Their Secondary Metabolites. Front. Microbiol. 2017, 8, 760. [CrossRef] [PubMed]

20. Niu, S.; Li, S.; Chen, Y.; Tian, X.; Zhang, H.; Zhang, G.; Zhang, W.; Yang, X.; Zhang, S.; Ju, J.; et al. Lobophorins $\mathrm{E}$ and F, new spirotetronate antibiotics from a South China sea-derived Streptomyces sp. SCSIO 01127. J. Antibiot. 2011, 64, 711-716. [CrossRef] [PubMed]

21. Song, Y.; Li, Q.; Liu, X.; Chen, Y.; Zhang, Y.; Sun, A.; Zhang, W.; Zhang, J.; Ju, J. Cyclic hexapeptides from the deep south China sea-derived Streptomyces scopuliridis SCSIO ZJ46 active against pathogenic gram-positive bacteria. J. Nat. Prod. 2014, 77, 1937-1941. [CrossRef] [PubMed]

22. Zhou, X.; Huang, H.B.; Li, J.; Song, Y.X.; Jiang, R.W.; Liu, J.; Zhang, S.; Hua, Y.; Ju, J. New antiinfective cycloheptadepsipeptide congeners and absolute stereochemistry from the deep sea-derived Streptomyces drozdowiczii SCSIO 10141. Tetrahedron 2014, 70, 7795-7801. [CrossRef] 
23. Song, Y.; Huang, H.; Chen, Y.; Ding, J.; Zhang, Y.; Sun, A.; Zhang, W.; Ju, J. Cytotoxic and antibacterial Marfuraquinocins from the deep South China sea-derived Streptomyces niveus SCSIO 3406. J. Nat. Prod. 2013, 76, 2263-2268. [CrossRef] [PubMed]

24. Kennedy, J.; Baker, P.; Piper, C.; Cotter, P.D.; Walsh, M.; Mooij, M.J.; Bourke, M.B.; Rea, M.C.; O'Conner, P.M.; Ross, R.P.; et al. Isolation and analysis of bacteria with antimicrobial activities from the marine sponge Haliclona simulans collected from Irish waters. Mar. Biotechnol. 2009, 11, 384-396. [CrossRef] [PubMed]

25. Kennedy, J.; Flemer, B.; Jackson, S.A.; Morrissey, J.P.; O'Gara, F.; Dobson, A.D.W. Evidence of a putative deep sea specific microbiome in marine sponges. PLoS ONE 2014, 9, e91092. [CrossRef] [PubMed]

26. Jackson, S.A.; Flemer, B.; McCann, A.; Morrissey, J.P.; O'Gara, F.; Dobson, A.D. Archaea appear to dominate the microbiome of Inflatella pellicula deep-sea sponges. PLoS ONE 2013, 8, e84438. [CrossRef] [PubMed]

27. Sims, G.E.; Jun, S.-R.; Wu, G.A.; Kim, S.-H. Alignment-free genome comparison with feature frequency profiles (FFP) and optimal resolutions. Proc. Natl. Acad. Sci. USA 2009, 106, 2677-2682. [CrossRef] [PubMed]

28. Wood, D.E.; Salzberg, S.L. Kraken: Ultrafast metagenomic sequence classification using exact alignments. Genome Biol. 2014, 15, R46. [CrossRef] [PubMed]

29. Weber, T.; Blin, K.; Duddela, S.; Krug, D.; Kim, H.U.; Bruccoleri, R.; Lee, S.Y.; Fischbach, M.A.; Muller, R.; Wohlleben, W.; et al. antiSMASH 3.0-A comprehensive resource for the genome mining of biosynthetic gene clusters. Nucleic Acids Res. 2015, 43, W237-W243. [CrossRef] [PubMed]

30. Medema, M.H.; Kottmann, R.; Yilmaz, P.; Cummings, M.; Biggins, J.B.; Blin, K.; de Bruijn, I.; Chooi, Y.H.; Claesen, J.; Coates, R.C.; et al. Minimum Information about a Biosynthetic Gene cluster. Nat. Chem. Biol. 2015, 11, 625-631. [CrossRef] [PubMed]

31. Borchert, E.; Jackson, S.A.; O'Gara, F.; Dobson, A.D.W. Diversity of natural product biosynthetic genes in the microbiome of the deep sea sponges Inflatella pellicula, Poecillastra compressa and Stelletta normani. Front. Microbiol. 2016, 7, 1027. [CrossRef] [PubMed]

32. Sandy, M.; Butler, A. Microbial iron acquisition: marine and terrestrial siderophores. Chem. Rev. 2009, 109, 4580-4595. [CrossRef] [PubMed]

33. Moore, J.K.; Braucher, O. Observations of dissolved iron concentrations in the world ocean: Implications and constraints for ocean biogeochemical models. Biogeosci. Discuss. 2007, 4, 1241-1277. [CrossRef]

34. Krasko, A.; Schröder, H.C.; Bate, R.L.; Grebenjuk, V.A.; Steffen, R.; Müller, I.M.; Müller, W.E.G. Iron induces proliferation and morphogenesis in primmorphs from the marine sponge Suberites domuncula. DNA Cell Biol. 2004, 21, 67-80. [CrossRef] [PubMed]

35. Mayzel, B.; Aizenberg, J.; Ilan, M. The elemental composition of Demospongiae from the Red Sea, Gulf of Aqaba. PLoS ONE 2014, 9, e99918. [CrossRef] [PubMed]

36. Tian, X.; Zhang, Z.; Yang, T.; Chen, M.; Li, J.; Chen, F.; Yang, J.; Li, W.; Zhang, B.; Zhang, Z.; et al. Comparative Genomics Analysis of Streptomyces Species Reveals Their Adaptation to the Marine Environment and Their Diversity at the Genomic Level. Front. Microbiol. 2016, 7, 998. [CrossRef] [PubMed]

37. Karen, R.; Mayzel, B.; Lavy, A.; Polishchuk, I.; Levy, D.; Fakra, S.C.; Pokroy, B.; Ilan, M. Sponge-associated bacteria mineralize arsenic and barium on intracellular vesicles. Nat. Commun. 2017, 8, 14393. [CrossRef] [PubMed]

38. Lane, D.J. 16S/23S rRNA sequencing. In Nucleic Acid Techniques in Bacterial Systematics; Stackebrandt, E., Goodfellow, M., Eds.; Wiley: New York, NY, USA, 1991; pp. 115-175.

39. Tamura, K.; Stecher, G.; Peterson, D.; Filipski, A.; Kumar, S. MEGA6: Molecular evolutionary genetics analysis version 6.0. Mol. Biol. Evol. 2013, 30, 2725-2729. [CrossRef] [PubMed]

40. Thompson, J.D.; Gibson, T.J.; Higgins, D.G. Multiple sequence alignment using ClustalW and ClustalX. Curr. Protoc. Bioinform. 2002. [CrossRef]

41. Nakhleh, L.; Jin, G.; Zhao, F.; Mellor-Crummey, J. Reconstructing Phylogenetic Networks Using Maximum Parsimony. In Proceedings of the 2005 IEEE Computational Systems Bioinformatics Conference, Stanford, CA, USA, 8-11 August 2005.

42. Cole, J.R.; Wang, Q.; Fish, J.A.; Chai, B.; McGarrell, D.M.; Sun, Y.; Brown, C.T.; Porras-Alfaro, A.; Kuske, C.R.; Tiedje, J.M. Ribosomal Database Project: Data and tools for high throughput rRNA analysis. Nucleic Acids Res. 2014, 42, D633-D642. [CrossRef] [PubMed]

43. Bibi, F. Diversity of antagonistic bacteria isolated from medicinal plant Peganum harmala L. Saudi J. Biol. Sci. 2017, 24, 1288-1293. [CrossRef] [PubMed] 
44. Bankevich, A.; Nurk, S.; Antipov, D.; Gurevich, A.; Dvorkin, M.; Kulikov, A.S.; Lesin, V.M.; Nikolenko, S.I.; Pham, S.; Prjibelski, A.D.; et al. SPAdes: A new genome assembly algorithm and its applications to single-cell sequencing. J. Comput. Biol. 2012, 19, 455-477. [CrossRef] [PubMed]

45. Contreras-Moreira, B.; Vinuesa, P. GET_HOMOLOGUES, a versatile software package or scalable and robust microbial pangenome analysis. Appl. Environ. Microbiol. 2013, 79, 7696-7701. [CrossRef] [PubMed] 